ARTICLE

\title{
Molecular insights into receptor binding of recent emerging SARS-CoV-2 variants
}

\author{
Pengcheng Han 1,2,3,17, Chao Su (10) 1,4,17, Yanfang Zhang 1,5,17, Chongzhi Bai ${ }^{6,7,17}$, Anqi Zheng ${ }^{1,8}$, Chengpeng Qiao1,

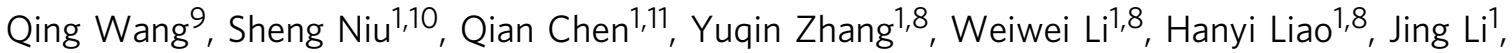 \\ Zengyuan Zhang ${ }^{1,8}$, Heecheol Cho ${ }^{2}$, Mengsu Yang ${ }^{4}$, Xiaoyu Rong 1,12, Yu Hu ${ }^{1,13}$, Niu Huang (1) 9,14,

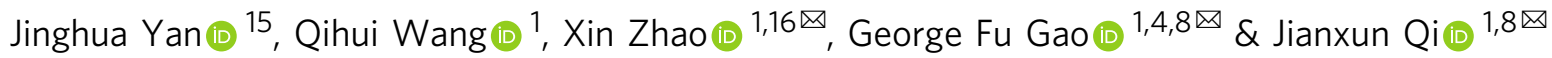

Multiple SARS-CoV-2 variants of concern (VOCs) have been emerging and some have been linked to an increase in case numbers globally. However, there is yet a lack of understanding of the molecular basis for the interactions between the human ACE2 (hACE2) receptor and these VOCs. Here we examined several VOCs including Alpha, Beta, and Gamma, and demonstrate that five variants receptor-binding domain (RBD) increased binding affinity for hACE2, and four variants pseudoviruses increased entry into susceptible cells. Crystal structures of hACE2-RBD complexes help identify the key residues facilitating changes in hACE2 binding affinity. Additionally, soluble hACE2 protein efficiently prevent most of the variants pseudoviruses. Our findings provide important molecular information and may help the development of novel therapeutic and prophylactic agents targeting these emerging mutants.

\footnotetext{
${ }^{1}$ CAS Key Laboratory of Pathogenic Microbiology and Immunology, Institute of Microbiology, Chinese Academy of Sciences, Beijing 100101, China. ${ }^{2}$ Department of Biomedical Engineering, Emory University, Atlanta, GA 30322, USA. ${ }^{3}$ College of Life Science and Technology, Southeast University, NanJing 210096, China. ${ }^{4}$ Department of Biomedical Sciences, City University of Hong Kong, Hong Kong 999077, China. ${ }^{5}$ Laboratory of Protein Engineering and Vaccines, Tianjin Institute of Industrial Biotechnology, Chinese Academy of Sciences, Tianjin 300308, China. ${ }^{6}$ Shanxi Academy of Advanced Research and Innovation, Taiyuan 030032, China. ${ }^{7}$ Central Laboratory, Shanxi Province Hospital of Traditional Chinese Medicine, Taiyuan 030012, China. ${ }^{8}$ University of the Chinese Academy of Sciences, Beijing 100049, China. ${ }^{9}$ National Institute of Biological Sciences, Beijing 102206, China. ${ }^{10}$ College of Veterinary Medicine, Shanxi Agricultural University, Jinzhong 030801, China. ${ }^{11}$ Institutes of Physical Science and Information Technology, Anhui University, Hefei 230601, China. ${ }^{12}$ School of Laboratory Medicine and Life Science, Wenzhou Medical University, Wenzhou 325035, China. ${ }^{13}$ School of Life Sciences, Division of Life Sciences and Medicine, University of Science and Technology of China, Hefei, Anhui 230026, China. ${ }^{14}$ Tsinghua Institute of Multidisciplinary Biomedical Research, Tsinghua University, Beijing 102206, China. ${ }^{15}$ CAS Key Laboratory of Microbial Physiological and Metabolic Engineering, Institute of Microbiology, Chinese Academy of Sciences, Beijing 100101, China. ${ }^{16}$ CAS Center for Influenza Research and Early-Warning (CASCIRE), Chinese Academy of Sciences, 100101

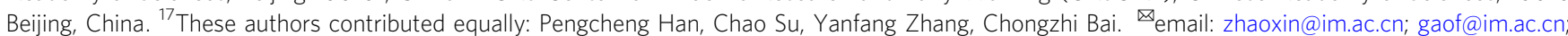
jxqi@im.ac.cn
} 
C oronavirus disease 2019 (COVID-19) which is caused by SARS-CoV-2 has rapidly been declared as pandemic since its identification in late December 20191,2. Up-to date, although tremendous efforts have led to the development of vaccine, combine with the preventive measures prescribed by the government around the world, the transmission of the virus among human are still going and numerous SARS-CoV-2 mutations with uncertain consequences for viral replication and transmission are being increasingly identified. The mutation of the spike (S) protein in SARS-CoV-2 has drawn wide concerns because $S$ proteins mediate viral entry via their interaction with the angiotensin-converting enzyme 2 (ACE2) receptor ${ }^{3,4}$ and are the major target for vaccine development. SARS-CoV-2 variant D614G in the S protein rapidly become dominant around the world ${ }^{5}$. Recently, several novel SARS-CoV-2 variants of concern (VOCs) carrying D614G mutation have been linked to an increased number of infections at a global scale (Supplementary Fig. 1).

VOC Alpha (B.1.1.7 lineage or 501Y.V1) emerged in the United Kingdom (UK) in September $2020^{6}$. It has been shown to be $75 \%$ more transmissible ${ }^{7}$ with a $43-90 \%$ higher reproduction rate $^{8}$ than earlier strains and has subsequently emerged as the dominant variant in the $\mathrm{UK}^{7,8}$. VOC Beta (B.1.351 lineage or 501Y.V2) was first identified in South Africa (SA) in October 2020, and has rapidly became the dominant strain in SA because of its high transmissibility ${ }^{9}$. The mutations of VOCs alter the viral fitness. The rapid spread of these variants of SARS-CoV-2 is likely due to the virus being more infectious, which means that small number virus particles can lead to infection. VOC Gamma (P.1 lineage or 501Y.V3) is a novel lineage tracing back to the B.1.1.28 lineage and was found to be circulating in Manaus, Brazil, in December 2020, with this isolate being identified in $42 \%$ of the specimens sequenced from this region in mid/late December ${ }^{10}$. And this Gamma variant was imported into Japan by travelers in early $2021^{11}$. N501Y in the receptor-binding domain (RBD) of S protein is a common substitution in Alpha, Beta, and Gamma strains, and is often found in combination with $\mathrm{E} 484 \mathrm{~K}$ and $\mathrm{K} 417 \mathrm{~N}$ (or K417T), with this combination described in the RBD of both the Beta and Gamma strains, respectively (Supplementary Fig. 1).

With the addition of variants circulating in humans, mutants have also been identified in susceptible farmed animals for fur. In Denmark, a SARS-CoV-2 variant referred to as the "Cluster 5" variant $^{12}$, and designated as Mink-Y453F in this study, was identified in farmed minks and subsequently found to be transmissible between minks and humans ${ }^{13}$. Besides, both F486L and N501T mutations have been found in SARS-CoV-2 isolates from minks, as well as humans ${ }^{14}$, and these strains were designated as Mink-F486L and Mink-N501T in this study, respectively (Supplementary Fig. 1). Given that residues at positions 417, 453, 486, and 501 are involved in the interactions between the RBD and human ACE2 (hACE2) ${ }^{4,15}$, it is important to understand the detailed binding of these novel variant RBDs to hACE2.

Here, we examined six different SARS-CoV-2 RBD variants, including Alpha, Beta, Gamma, Mink-Y453F, Mink-F486L, and Mink-N501T. We found that these dominant mutations result in enhanced binding affinity to hACE2 receptor except for MinkF486L, and that four variants pseudovirus particles except for Gamma and Mink-F486L show enhancement of viral entry to human cells. Analysis of molecular features of crystal structures revealed that replacements of key amino acids change bonding forces in the interaction interface. In addition, we demonstrate that the soluble hACE2 protein efficiently inhibit most of SARSCoV-2 variants infections. Taken together, these data highlight the entry of new emerging variants and will provide a useful information to develop antiviral drugs against SARS-CoV-2.

\section{Results}

The binding characteristic of SARS-CoV-2 variants RBD to hACE2. The binding of RBD to the receptor hACE2 is an essential step for virus to entry cell, thus we first explored if the binding of six SARS-CoV-2 variant (including Alpha, Beta, Gamma, Mink-Y453F, Mink-F486L and Mink-N501T) RBDs to hACE2 were changed in comparison with wild-type (WT) SARSCoV-2 (the first strain of SARS-CoV-2 which was isolated from a clinical patient on Jan 6, 2020, GISAID: EPI_ISL_402119) RBD. The SARS-CoV-2 WT RBD and variant RBDs were purified and then evaluated for hACE2 binding using flow cytometry (FACS) (Supplementary Fig. 2). SARS-CoV-2 Alpha, Beta, and Gamma, as well as Mink-Y453F and Mink-N501T RBDs, bound to the hACE2-expressing cells with higher affinity than WT RBD, with $33.4 \%$ positive cells (for WT RBD) versus $82.7 \%$ (for Alpha RBD), 48.1\% (for Beta RBD), $57.7 \%$ (for Gamma RBD), $84.7 \%$ (for Mink-Y453F RBD), and 76.8\% (for Mink-N501T RBD), respectively (Fig. 1). While Mink-F486L RBD bound to fewer hACE2expressing cells with a $1.65 \%$ positivity rate (Fig. 1).

To better understand the interactions between SARS-CoV-2 variant RBDs and hACE2, we measured their binding affinity using surface plasmon resonance (SPR). Mouse Fc (mFc) tagged hACE2 was captured in a CM5 chip that pre-immobilized with anti-mFc antibody, and the serially diluted variant RBDs were flowed through the chip. As shown in Fig. 2, SARS-CoV-2 WT RBD protein bound to hACE2 with an equilibrium dissociation constant $\left(K_{\mathrm{D}}\right)$ of $\sim 26.34 \mathrm{nM}$ (Fig. $2 \mathrm{a}$ ), which is similar to the previous results ${ }^{16,17}$. Alpha RBD, Beta RBD, Gamma RBD, MinkY453F RBD, and Mink-N501T RBD displayed higher affinities to hACE2 than WT RBD, with $\sim 7, \sim 3, \sim 5, \sim 8$, and $\sim 4$-fold increase in binding strength, respectively (Fig. $2 \mathrm{~b}-\mathrm{f}$ ). Notably, both Beta RBD and Gamma RBD contain two more mutated residues than Alpha RBD, but displayed a little lower binding affinities for hACE2 than Alpha RBD. In order to understand which residues in Beta RBD and Gamma RBD contribute to the lower affinities, three single mutations (including K417N, K417T, and E484K), and three double mutations (containing N501Y/E484K, N501Y/ $\mathrm{K} 417 \mathrm{~N}$, and N501Y/K417T) were prepared and used to measure the binding affinities for hACE2. Both K417N and K417T mutations in RBD decreased $\sim 2$ fold affinity for hACE2, while E484K mutation exerted little effect (Supplementary Fig. 3). Similarly, both N501Y/K417N RBD and N501Y/K417T RBD also displayed $\sim 2$ fold lower affinity than N501Y RBD, but N501Y/ E484K did not (Supplementary Fig. 3). Although both N501Y and N501T strengthened the interactions between RBD and hACE2, N501Y exhibits higher affinity than N501T. In contrast, MinkF486L RBD bound to hACE2 with $\sim 4$-fold lower binding affinity than WT RBD (Fig. 2g). These results were consistent with the FACS assays. In a word, five variant (Alpha, Beta, Gamma, MinkY453F, and Mink-N501T) RBDs but not Mink-F486L RBD, increased association with hACE2.

Current data indicate that Mink-Y453F, Mink-N501T, and MinkF486L emerged in farmed minks, when we evaluated their RBDs binding affinity for mink ACE2 (miACE2, Neovison vison ${ }^{13,18}$ ), we noted that WT RBD bound to miACE2 with $\sim 8.16 \mu \mathrm{M}$ affinity, which was $\sim 310$-fold lower than the affinity of WT RBD to hACE2 (Fig. 2h). In addition, Mink-Y453F RBD, Mink-N501T RBD, and Mink-F486L RBD exhibited much higher binding affinity for miACE2 than WT RBD with $\sim 21, \sim 9$, and $\sim 15$ fold, respectively (Fig. 2i-k). This provides some molecular evidence to explain why the mutant strains efficiently transmitted among minks.

The molecular basis of hACE2 bound to SARS-CoV-2 variants RBD. To further elucidate the molecular mechanism underlying the binding of these SARS-CoV-2 variant RBDs to hACE2, we 

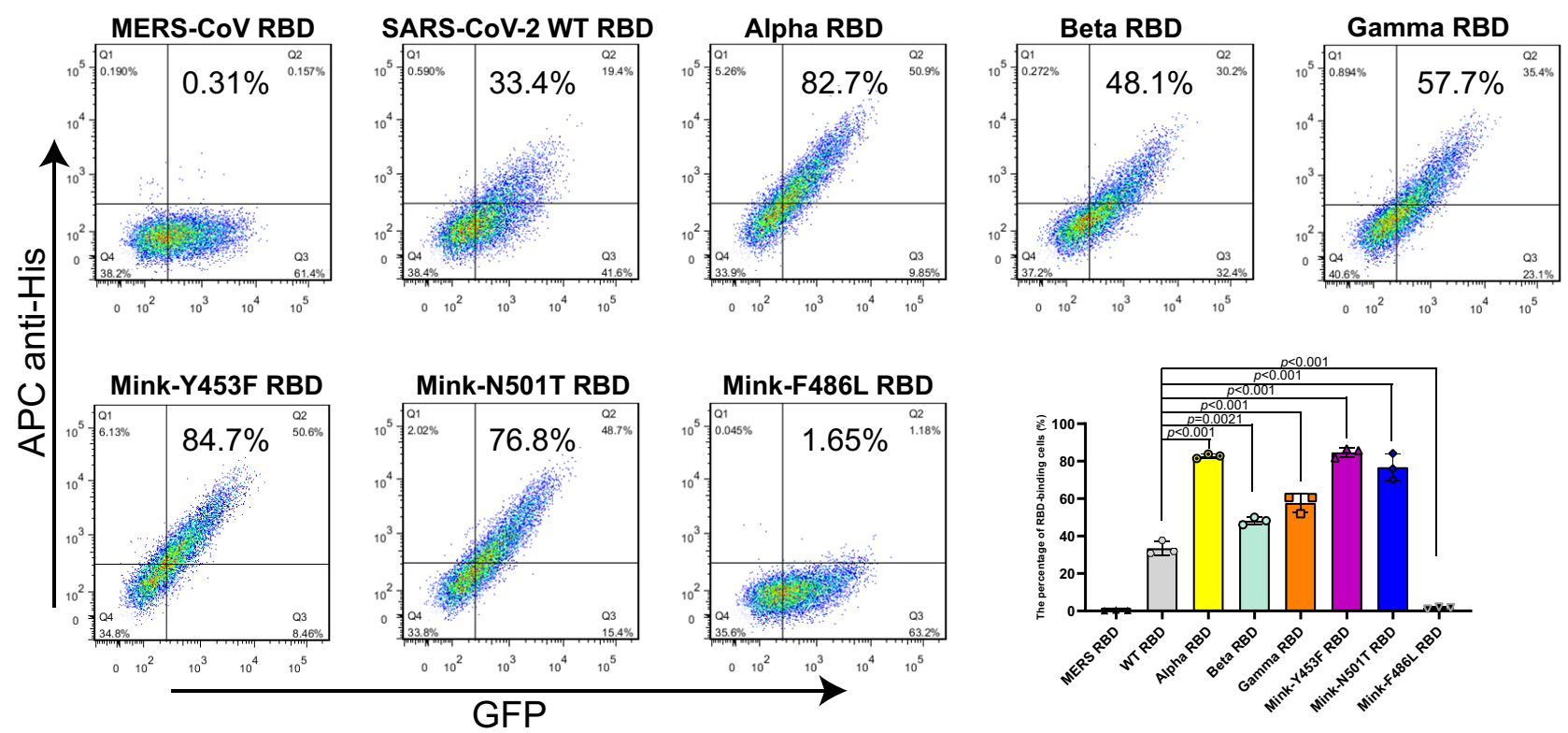

Fig. 1 Characterization of binding between different RBDs and hACE2 using flow cytometry. BHK cells stably expressing GFP and hACE2 were incubated with His-tagged MERS-CoV RBD, SARS-CoV-2 WT RBD, Alpha RBD, Beta RBD, Gamma RBD, Mink-Y453F RBD, Mink-N501T RBD, and Mink-F486L RBD, respectively. APC anti-His antibodies were used to detect the His-tagged protein binding to the cells. Representative results from three experiments are shown. The mean \pm SD percentages of RBD-binding cells in the three experiments are shown in the right lower bar chart. Statistical significance was analyzed using one-way ANOVA with a Tukey's multiple comparison test for multiple groups.

prepared for RBD-hACE2 complexes and obtained five crystal structures, namely Alpha RBD-hACE2, Beta RBD-hACE2, Gamma RBD-hACE2, Mink-Y453F RBD-hACE2, and MinkF486L RBD-hACE2 at a resolution of $2.9 \AA$, $2.6 \AA, 2.8 \AA, 2.4 \AA$, and $2.7 \AA$, respectively (Table $\mathrm{S} 1$ ). Each complex structure was comprised of one copy of the RBD-hACE2 molecule in one asymmetric unit. The overall structure of Alpha RBD-hACE2, Beta RBD-hACE2, Gamma RBD-hACE2, Mink-Y453F RBDhACE2, and Mink-F486L RBD-hACE2 were similar to the WT RBD-hACE2 structure, with a root-mean-square deviation (RMSD) of $0.196 \AA$ (for $736 \mathrm{Ca}$ atoms), $0.109 \AA$ (for $725 \mathrm{Ca}$ atoms), $0.198 \AA$ (for $743 \mathrm{Ca}$ atoms), $0.158 \AA$ (for $712 \mathrm{Ca}$ atoms) and $0.150 \AA$ (for $763 \mathrm{Ca}$ atoms), respectively (Supplementary Fig. 4), when compared with the WT RBD-hACE2 (PDB: 6LZG) structure.

For the detailed mutated positions (Supplementary Fig. 5), in Alpha RBD-hACE2, Beta RBD-hACE2, and Gamma RBDhACE2 structures, N501 is located in a loop structure and could be replaced by a Y without creating folding problems, it seems that the N501Y does not induce a large conformational change. Some very weak hydrogen bonds are possible between N501 and ACE2, but the phenyl of the Y501 side chain could make many new favorable nonbonded interactions with hACE2, i.e., a cation$\pi$ interaction with hACE2 K353 and a $\pi-\pi$ stacking interaction with hACE2 Y41 (Fig. 3a-c). These noncovalent interactions in Y501 variant are stronger as compared to the WT type. Thus, Y501 significantly increased the interaction between RBD and hACE2 compared to N501, so the N501Y replacement in this region of the interface should be favorable for the interaction with hACE2. These results were consistent with cryo-electron microscopy structures of the N501Y SARS-CoV-2 spike protein in complex with $\mathrm{ACE} 2^{19}$. In addition, the E484K substitutions would seem neutral or even unfavorable because it is far away from the RBD-interacting residues of hACE2 (Fig. 3a, b). This can also be confirmed by the results of the binding affinity of hACE2 with E484K single or double mutation constructions (Supplementary Fig. 3). Both K417N mutation in Beta RBD and
K417T mutation in Gamma RBD destroyed the salt bridge formed by K417 and hACE2 D30 (Fig. 3a, b). Therefore, Beta RBD and Gamma RBD exhibited a higher binding affinity for hACE2 than WT RBD, but a little lower than Alpha RBD, consistent with the SPR results. In the Mink-F486L RBDhACE2 structure, the mutation at F486L impairs the $\pi-\pi$ stacking interaction formed by RBD F486 and hACE2 Y83 (Fig. 3d), resulting in the decreased interaction between Mink-F486L RBD and hACE2. We also predicted the structure of miACE2 and compared it with both Mink-F486L RBD-hACE2 and WT RBDhACE2 structures. The residue T82 in miACE2 was clashed with the phenyl group of RBD F486 but not L486 (Supplementary Fig. 6). Thus, F486L mutation may be helpful for the interaction of SARS-CoV-2 RBD with miACE2.

In the Mink-Y453F RBD-hACE2 structure, the carbonyl group of hACE2 D30 forms a hydrogen bond with N 81 in hACE2 H34, leading to the $\mathrm{C} \delta 2$ from $\mathrm{H} 34$ forming a hydrophobic interaction with the phenyl group of RBD F453 (Fig. 3e). Thus, when compared with the hydrophilic hydroxyphenyl group of WT RBD Y453, the phenyl group at this residue (F453) shifts toward hACE2 H34 by $0.4 \AA$ (Fig. 3e). In addition, the molecular dynamics (MD) simulations and molecular mechanics/PoissonBoltzmann surface area (MM/PBSA) evaluations revealed that the binding energy of Mink-Y453F RBD-hACE2 was significantly more favorable than WT RBD-hACE2 (Supplementary Table 2), and $\mathrm{F} 453$ showed better binding energy than $\mathrm{Y} 453\left(\Delta \Delta \mathrm{E}_{\text {Binding }}=\right.$ $\left.-1.03 \mathrm{kcal} \cdot \mathrm{mol}^{-1}\right)$, with the major contribution from the desolvation term (Supplementary Table 3). In addition, several adjacent residues were also shown to make favorable energy contributions to this binding when compared to the WT RBDhACE2 complex, including residues R403 and K417 in the RBD, and D30 in the hACE2 (Supplementary Table 3). Moreover, the number of hydrogen bonds in the Mink-Y453F RBD-hACE2 increased, by one, when compared to WT RBD-hACE2. This includes hydrogen bond between hACE2 Y83 and Mink-Y453F RBD Y489 (Fig. 3f and Supplementary Fig. 7). Thus, the complex structure reported here provides the molecular explanation why 
a

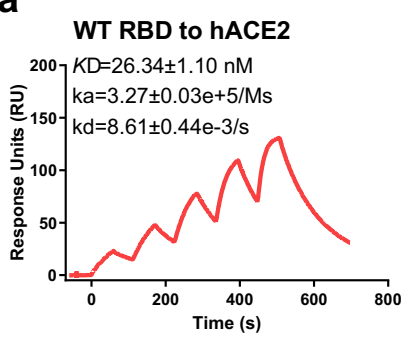

e

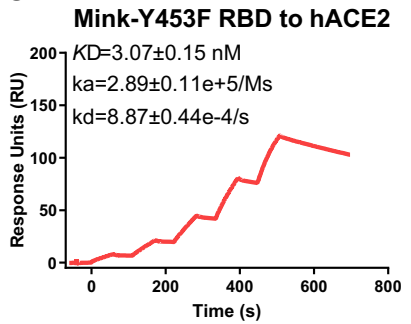

h

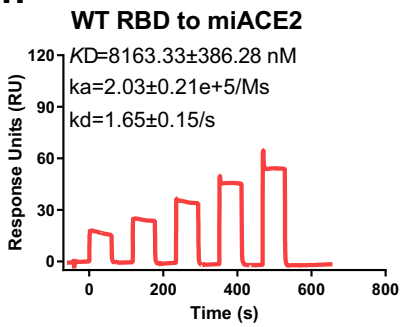

b

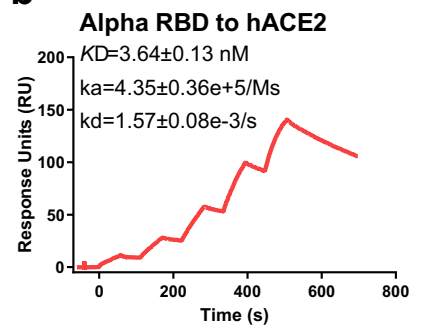

f

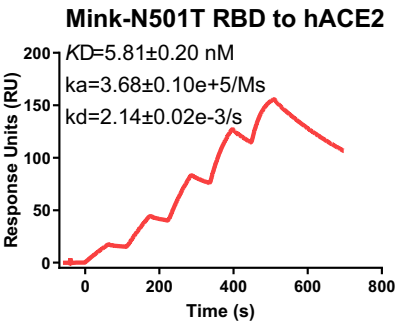

i

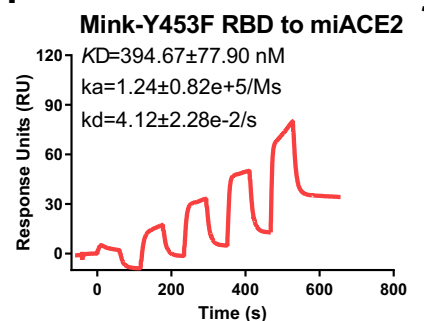

C

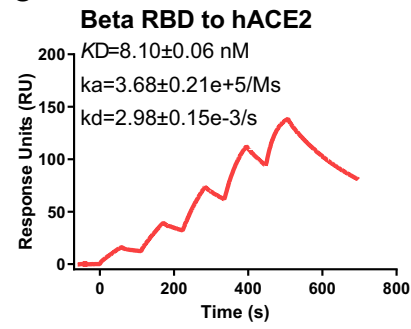

g

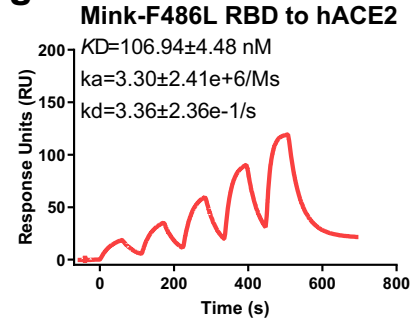

j

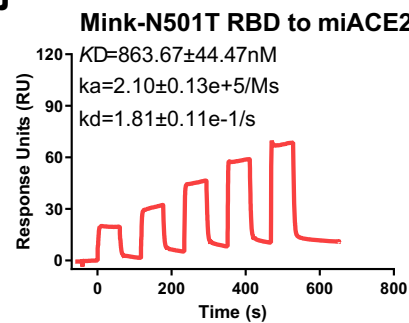

d

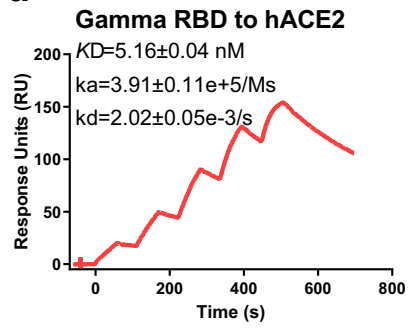

\section{k}

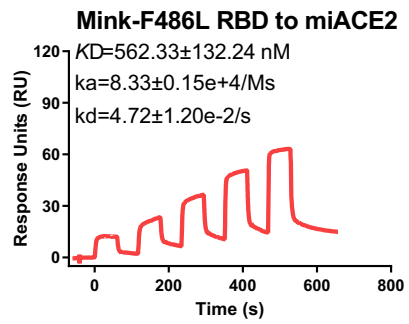

Fig. 2 Binding affinity of SARS-CoV-2 RBDs to ACE2, characterized by SPR. a-k Mouse Fc (mFc)-fused hACE2 or miACE2 in the supernatant was captured in the CM5 chip via its interaction with the pre-immobilized anti-mFc antibody. Various concentrations of SARS-CoV-2 WT RBD (a), Alpha RBD (b), Beta RBD (c), Gamma RBD (d), Mink-Y453F RBD (e), Mink-N501T RBD (f), and Mink-F486L RBD (g) protein were used to evaluate their binding affinity for hACE2. Serially diluted WT RBD (h), Mink-Y453F RBD (i), Mink-N501T RBD (j), and Mink-F486L RBD (k) protein were measured the binding to miACE2. $K_{\mathrm{D}}, \mathrm{ka}$, and $\mathrm{kd}$ values are all recorded and the representative results from three experiments are shown. The data are presented as the mean \pm SEM of three independent replicates $(n=3)$.

Mink-Y453F RBD demonstrated an increased binding affinity for hACE2 when compared to WT RBD, which is observed in this and previous studies ${ }^{20,21}$.

The transduction of SARS-CoV-2 variants pseudoviruses engaged by ACE2. Considering that the binding affinity of SARSCoV-2 variants RBD to hACE2 are changed, we further tested the potential influence of the SARS-CoV-2 variants on cellular infection using pseudovirus transduction. The same amount of pseudovirus that incorporate into the various SARS-CoV-2 variants $S$ protein were infected hACE2-positive Huh7 cells and the GFP of pseudovirus was quantified by FACS for the transduction efficiency. As we saw in the binding affinity assays, pseudovirus particles of Alpha, Beta, Mink-N501T, and Mink-Y453F, but not Mink-F486L, showed increased transduction efficiency when compared to the D614G strain in Huh7 cells (Fig. 4a-f, and h). However, inconsistent with binding affinity, Gamma pseudovirus displayed the similar transduction efficiency with the D614G pseudovirus (Fig. 4g, h). In order to exclude the influence of binding of RBD to hACE2 on the different transduction efficiency between Beta and Gamma, N417T mutation was introduced to Beta S protein, which was designated as Beta-N417T that contains the same RBD sequence as Gamma. The transduction efficiency of Beta-N417T pseudovirus was similar to Beta pseudovirus and higher than Gamma pseudovirus (Supplementary Fig. 8). It suggested that other substitutions outside of the RBD also contribute to the change in transduction efficiency. Taken together, four out of six variants exhibited increased transduction efficiency.

The neutralization of SARS-CoV-2 variants by soluble hACE2. Considering that both Beta and Gamma were resistant to the antibodies-mediated neutralization ${ }^{22,23}$, soluble hACE2 was a potential therapy for COVID-19, especially under the background of higher affinity of SARS-CoV-2 variants to hACE2. We then tested the neutralization of soluble hACE2 protein to SARS-CoV2 variants, and observed that soluble hACE2 protein efficiently prevent SARS-CoV-2 variants pseudoviruses from the entry into susceptible cells, and the inhibitory effect of soluble hACE2 protein to the SARS-CoV-2 variant pseudoviruses, with the exception of Mink-F486L, is higher than the SARS-CoV-2, especially Gamma (Fig. 5). It highlighted the importance of hACE2 targeted SARS-CoV-2 variants.

\section{Discussion}

The deep mutational scanning suggests that single residue mutation $\mathrm{K} 417 \mathrm{~N}$ or $\mathrm{K} 417 \mathrm{~T}$ is likely to have minimal effect on the binding to hACE2, and that the E484K mutation may predictably enhance the binding ${ }^{21}$, while in this study the structures of Beta RBD-hACE2 and Gamma RBD-hACE2 indicated the mutations, K417N and K417T, decreased the RBD-hACE2 interactions while the mutation $\mathrm{E} 484 \mathrm{~K}$ displayed little impact. The structural information was confirmed by the SPR results that the single mutation (K417N or K417T) or double mutation (N501Y/K417N 


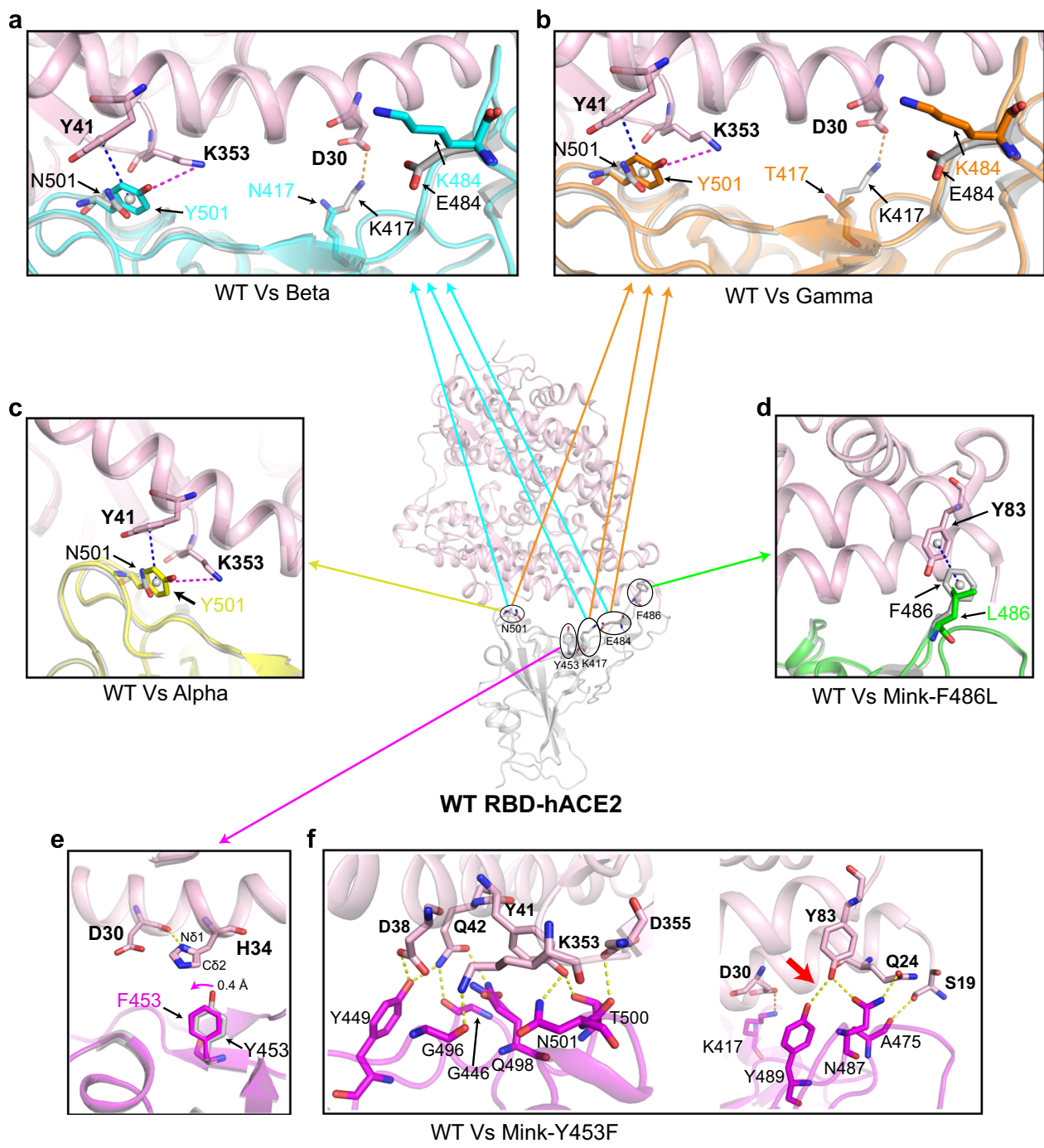

Fig. 3 Structural comparison of WT RBD-hACE2 and each SARS-CoV-2 variants RBD-hACE2. a-e The WT RBD-hACE2 structure (PDB: 6LZG) is shown in the center. Superimposition of WT RBD-hACE2 and each variant RBD-hACE2 (including Beta RBD-hACE2 (a), Gamma RBD-hACE2 (b), Alpha RBDhACE2 (c), Mink-F486L RBD-hACE2 (d), Mink-Y453F RBD-hACE2 (e)) are shown in each surrounding panel. In each structure the hACE2 is colored in light pink. SARS-CoV-2 WT RBD, Beta RBD, Gamma RBD, Alpha RBD, Mink-F486L RBD, and Mink-Y453F RBD are colored in gray, cyan, orange, yellow, green, and magenta, respectively. The key contact residues are shown as stick structures and labeled appropriately. The cation- $\pi$ interaction, $\pi-\pi$ stacking interaction, salt bridge, and hydrogen bonds are colored in magenta, blue, orange, and yellow, respectively. Hydrogen bond interactions were analyzed at a cutoff of $3.5 \AA$. $\mathbf{f}$ The detailed hydrogen bonds between the Mink-Y453F RBD and hACE2 are shown.

or N501Y/K417T) RBD displayed a lower binding affinity for hACE2 than WT RBD or RBD N501Y, but E484K or N501Y/ E484K did not. Interestingly, the decreased impact caused by K417N or K417T could be quantitatively counteracted by the increased binding effect associated with the N501Y mutation.

Although we and other groups found a higher binding affinity of Mink-Y453F RBD to hACE2 compared with WT RBD ${ }^{20,21}$, it has been reported that the OH group of RBD Y453 is involved in direct interaction with hACE2 H34 in the cryo-electron microscopy (cryo-EM) complex structure of full-length hACE2 bound to RBD and $\mathrm{B}^{0} \mathrm{AT} 1$ (with the resolution of $\left.2.9 \AA\right)^{24}$. Interestingly, this interaction was not observed in the crystal complex structures of the hACE2 ectodomain bound to RBD (PDB: 6LZG and 6M0J) (with the resolution of $2.5 \AA$ ) or bound to Mink-Y453F RBD in this study (with the resolution of $2.4 \AA$ ). This discrepancy may be the result of the low resolution for hACE2 residue H34 within the RBD-hACE2- $\mathrm{B}^{0} \mathrm{AT} 1$ structure.

The changes in binding affinity and viral membrane fusion process may impact viral entry in the host. For instance, the
N501Y mutation strengthens the binding affinity for mouse ACE2 and supports the adaptation of SARS-CoV-2 to mice in vitro and in vivo ${ }^{25}$. Corresponding with the binding affinity, more efficient transduction efficiency of pseudovirus particles of Alpha and Beta than D614G strain were observed in susceptive cells, although our assays were limited to pseudovirus viruses. It accounted at least in part for the higher transmissibility of Alpha $^{7}$, and the fact that the frequency of viruses containing Alpha and Beta $S$ sequences grew rapidly among the sequences available from the GISAID Initiative database (Supplementary Fig. 9a, b). In addition, the D614, which is outside RBD, located in the S1 subunit of the WT protein. The hydrogen bond formed by D614 residue and T859 residue in the adjacent propolymer S2 region disappeared after the mutation from $D$ to $G$, which improved the cleavage efficiency of furin protease and made S1 protein more easily fall off from S2 protein, promoting the viral membrane fusion process, suggesting that D614G mutation increases entry efficiency ${ }^{26}$. Moreover, the P681R mutation in the spike protein, facilitates the spike protein cleavage and enhances 


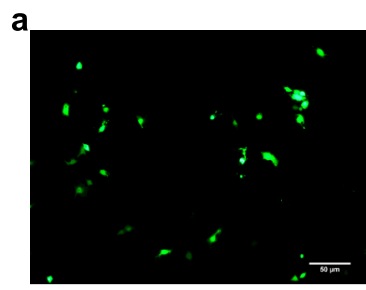

D614G

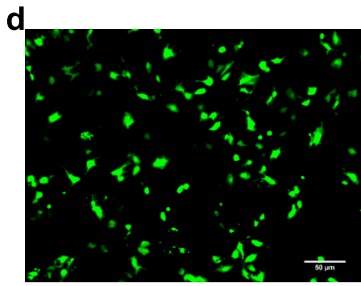

Mink-N501T

g

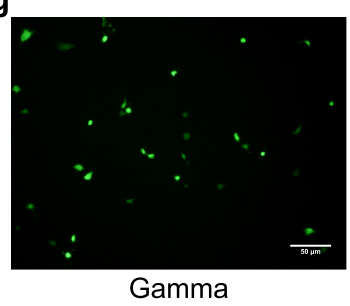

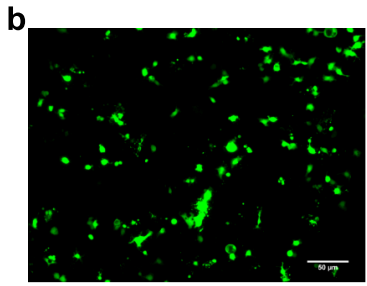

Alpha

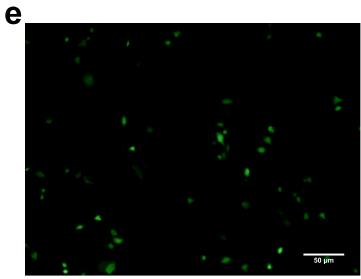

Mink-Y453F

h

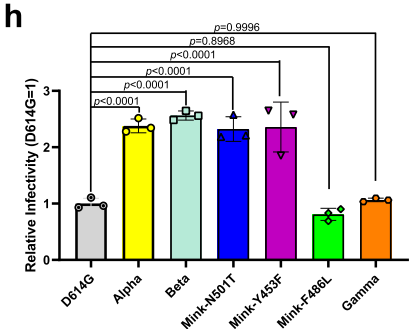

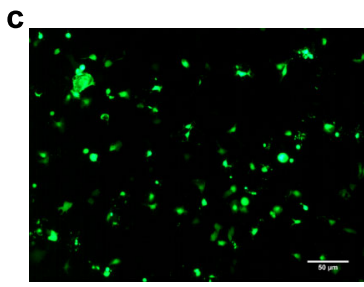

Beta

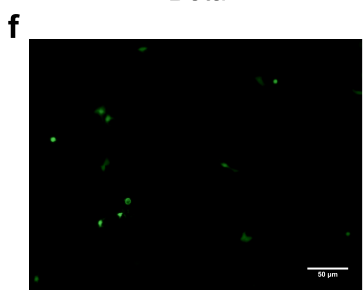

Mink-F486L

Fig. 4 Entry of SARS-CoV-2 variant pseudoviruses into Huh7 cells. a-g The SARS-CoV-2 D614G (a), Alpha (b), Beta (c), Mink-N501T (d), Mink-Y453F (e), Mink-F486L (f), and Gamma (g) pseudoviruses' entry into Huh7 cells as evidenced by GFP expression in transduced cells. The representative results from three experiments are shown. $\mathbf{h}$ The GFP-positive cells were quantified using FACS and representative results from three experiments are shown. The values indicate the mean of the three experiments and the bar suggest the SD. Relative infectivity was normalized against that of the D614G pseudovirus. Statistical significance was analyzed using a one-way ANOVA with Tukey's multiple comparison test for multiple groups.

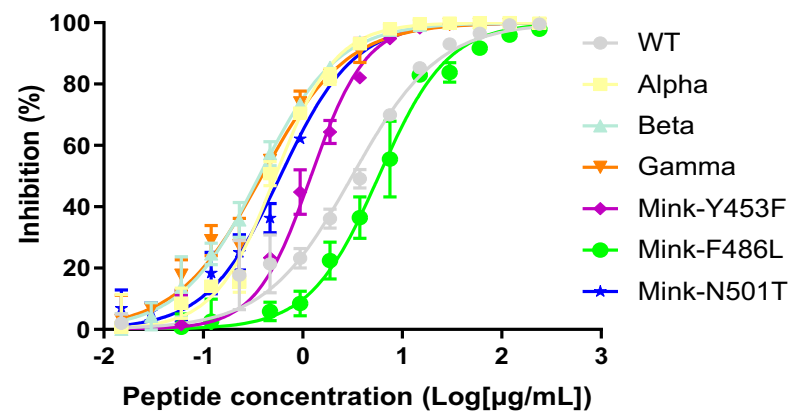

\begin{tabular}{lrc}
\hline & $\mathrm{EC}_{\mathbf{5 0}}(\boldsymbol{\mu g} / \mathrm{mL})$ & Folds change \\
\hline WT & $5.61 \pm 2.43$ & \\
Alpha & $0.66 \pm 0.24$ & 8.50 \\
Beta & $1.42 \pm 0.87$ & 3.95 \\
Gamma & $0.22 \pm 0.10$ & 25.50 \\
Mink-Y453F & $1.05 \pm 0.33$ & 5.34 \\
Mink-F486L & $15.40 \pm 5.16$ & 0.36 \\
Mink-N501T & $1.04 \pm 0.23$ & 5.39 \\
\hline
\end{tabular}

Fig. 5 Neutralization of SARS-CoV-2 variants by hACE2. The $\mathrm{EC}_{50}$ values and folds change values are shown on the right panel. The folds change was normalized to $\mathrm{WT}$, and the $\mathrm{EC}_{50}$ values are presented as the mean $\pm \mathrm{SEM}$ of three independent replicates $(n=3)$. The representative results from three experiments are shown.

the efficacy of viral fusion and further accelerates its speed of $\operatorname{action}^{27}$.

SARS-CoV-2 may acquire adaptive mutations that ensure efficient viral replication and transmission in other species, for example, by optimizing the interaction with host ACE2. The RBDs of three mink-origin variants: Mink-Y453F, Mink-F486L, and Mink-N501T, displayed a higher binding capacity to mink ACE2. These mutations may be adapted for the efficient use of mink ACE2 for entry. Thus, the number of both Mink-Y453F and Mink-F486L S sequences grew rapidly before the beginning of November 2020 (Supplementary Fig. 9c, d). However, MinkN501T, whose binding affinity for hACE2, transduction efficiency, and frequency of S sequences (Supplementary Fig. 9e) were similar with both Alpha and Beta, was likely to adapt to transmission among humans, but Mink-F486L not. This may partly explain why Mink-F486L did not efficiently transmit to humans and suddenly disappeared following the implementation of the mink cull policy in the Netherlands and Denmark (Supplementary Fig. 9d). While, increasing human samples were detected to carry N501T (Supplementary Fig. 9e). However, Mink-N501T has not drawn enough attention. Considering SARS-CoV-2 has been detected in farmed minks in ten countries in Europe and North America ${ }^{28}$, our results suggest it should be evaluated in more detail in the future, especially in people who live or work on mink farms and are close to mink.

Although Mink-Y453F showed a higher level of transduction efficiency than Gamma, the frequency of their $S$ sequences (GISAID) displayed a reverse tendency (Supplementary Fig. 9c, f). 
With the addition to the possibility of sampling bias and detection limitation in the relevant countries and regions, the reverse tendency suggested that other effects besides of viral entry brought out by the mutations, such as immune escape, probably impacted on the transmissibility of SARS-CoV-2 variants. It was supportively reported that Gamma escaped from vaccine-elicited neutralizing responses, but not Mink-Y453F 22 .

The result of live-virus neutralization assay showed that the Beta variant was poorly cross-neutralized by plasma from individuals with first-wave infections (did not contain the mutations associated with Beta) in South Africa ${ }^{29}$. The result of pseudoparticles infection indicated that entry of the Beta and Gamma variants were partially (Casirivimab) or fully (Bamlanivimab) resistant to antibodies used for COVID-19 treatment ${ }^{23}$. These results suggest that these variants may escape from neutralization by antibodies ${ }^{23}$ and convalescent plasma ${ }^{29,30}$. It is necessary to develop other therapeutic strategies for these variants. A potential drug-soluble human ACE2 protein was proved to reduce viral load in Vero E6 cells by $1000-5000-$ fold $^{31}$, which has already conducted a two-part clinical phase 2 trial $^{32,33}$. Soluble hACE2 protein displayed a more efficient inhibition to most SARS-CoV2 variants, due to the higher binding affinity to hACE2. Thus, the engineered ACE2 that increased the binding affinity for SARS$\mathrm{CoV}-2$ could serve as a potential therapeutic against SARS-CoV-2 variants.

As more SARS-CoV-2 variants continue to emerge and the major SARS-CoV-2 variants continue to spread, characterization of the hACE2-binding affinity and transduction efficiency of SARS-CoV-2 variants will help us understand SARS-CoV-2 transmission. The molecular features of variant RBDs binding to hACE2 provides valuable information helping us understand the entry mechanism of SARS-CoV-2 variants and aiding in the development of novel vaccines and specific drugs that target the SARS-CoV-2 entry process.

\section{Methods}

Cells. Expi293F cells (Gibco) were cultured at $37^{\circ} \mathrm{C}$ in SMM 293-TII Expression Medium with $5 \% \mathrm{CO}_{2}$ in a shaking incubator $(140 \mathrm{rpm})$. Sf 9 cells (Invitrogen) and High Five cells (Invitrogen) were cultured at $27^{\circ} \mathrm{C}$ in Insect-XPRESS medium (LONZA) in a shaking incubator $(120 \sim 130 \mathrm{rpm})$.

HEK293T (ATCC), BHK21 (ATCC) and Huh7 cells (3111C0001CCC000679) were cultured at $37^{\circ} \mathrm{C}$ in Dulbecco's Modified Eagle medium (DMEM) supplemented $10 \%$ fetal bovine serum (FBS) at $5 \% \mathrm{CO}_{2}$

Gene cloning, expression and protein purification. The coding sequence of hACE2-mFc (residues 1-740, GenBank: NP_001358344) or miACE2-mFc (residues 1-740, GenBank: QPL12211) were cloned into pCAGGS vector (MiaoLing-

Plasmid). The plasmids were transiently transfected into HEK293T cells using PEI and then, $48 \mathrm{~h}$ later, the cell supernatants were collected, concentrated and used in the SPR assays.

The DNA sequence encoding hACE2 (residues 19-615, GenBank: NP_001358344) was inserted into the Baculovirus transfection vector pFastBacl (Invitrogen) using the EcoRI and XhoI restriction sites. The gp67 signal peptide sequence was added to the N-terminus of the hACE2 gene for protein secretion, and the Hexa-His tag sequence was added to the C-terminus of the

hACE2 sequence for protein purification. The hACE2 protein was expressed using the Bac-to-Bac Baculovirus expression system and used for crystallization. The pFastBac1-hACE2 plasmids were transformed into DH10Bac E. coli to produce recombinant bacmids. Transfection of the bacmids using FuGENE 6 Transfection Reagent (Promega) and virus amplification were carried out in Sf9 cells, and the proteins were expressed in High Five cells. The supernatants were collected $48 \mathrm{~h}$ post-infection.

The DNA sequences encoding hACE2 (residues 1-740, GenBank: NP_001358344) were cloned into the pCAGGS vector with Hexa-His tag at the $\mathrm{C}$-terminus. The DNA sequences encoding SARS-CoV-2 WT RBD (spike residues 319-541, GISAID: EPI_ISL_402119) or MERS-CoV RBD (spike residues 367-606, GenBank: JX869050) were inserted into the pCAGGS vector with IL10 signal peptide sequence at the $\mathrm{N}$-terminus and the Hexa-His tag at the $\mathrm{C}$-terminus. The SARS-CoV-2 variant RBD plasmids (including Alpha RBD, Beta RBD, Gamma RBD, Mink-Y453F RBD, Mink-F486L RBD, Mink-N501T RBD, RBD N501Y/ E484K, RBD N501Y/K417N, RBD N501Y/K417T, RBD K417N, RBD K417T, and RBD E484K) were constructed via site-directed mutagenesis using the Mut Express
II Fast Mutagenesis Kit V2 (Vazyme). The recombinant RBD and hACE2 (used for pseudovirus neutralization assays) proteins were expressed in Expi293F cells after plasmid transfection using Sinofection Transfection Reagent (Sino Biological). The supernatants were collected 5 days post-transfection.

The supernatants containing hACE2 or RBD proteins were purified via affinity chromatography using a HisTrap HP $5 \mathrm{~mL}$ column (GE healthcare) and the target proteins were eluted in an elution buffer composed of $20 \mathrm{mM}$ Tris $(\mathrm{pH} \mathrm{8.0)}$ ), $150 \mathrm{mM} \mathrm{NaCl}$, and $300 \mathrm{mM}$ imidazole. The samples were then purified using gelfiltration chromatography on a HiLoad 16/600 Superdex 200PG column (GE healthcare) in a buffer containing $20 \mathrm{mM}$ Tris ( $\mathrm{pH} 8.0)$ and $150 \mathrm{mM} \mathrm{NaCl}$

Complex preparation and crystallization. Purified hACE2 and each SARS-CoV-2 variant RBD protein (including Alpha RBD, Beta RBD, Gamma RBD, Mink-Y453F $\mathrm{RBD}$, and Mink-F486L RBD) were mixed and incubated on ice for $2 \mathrm{~h}$. The mixture was then purified on HiLoad 16/600 Superdex 200PG column in a buffer containing $20 \mathrm{mM}$ Tris ( $\mathrm{pH}$ 8.0) and $50 \mathrm{mM} \mathrm{NaCl}$. The SARS-CoV-2 variant RBD-hACE2 complex proteins were then concentrated to $15 \mathrm{mg} / \mathrm{mL}$ for crystallization. All crystallizations were performed using a vapor-diffusion sitting-drop method with $0.8 \mu \mathrm{L}$ protein mixing with $0.8 \mu \mathrm{L}$ reservoir solution at $18^{\circ} \mathrm{C}$. High-quality crystals for both the Beta RBD-hACE2 and Gamma RBD-hACE2 complexes were obtained when using $0.1 \mathrm{M}$ MES ( $\mathrm{pH}$ 6.5), 12\% w/v PEG 20000 at a concentration of $15 \mathrm{mg} / \mathrm{mL}$ at $18^{\circ} \mathrm{C}$. Complex crystals of Alpha RBD-hACE2, Mink-Y453F RBDhACE2, and Mink-F486L RBD-hACE2 were grown in $0.1 \mathrm{M} \mathrm{MES} \mathrm{(pH} \mathrm{6.5),} 10 \% \mathrm{w}$ / v PEG $5000 \mathrm{MME}, 12 \% \mathrm{v} / \mathrm{vl}$-Propanol at a concentration of $15 \mathrm{mg} / \mathrm{mL}$ at $18^{\circ} \mathrm{C}$.

Data collection and structure determination. Prior to collecting diffraction data, all crystals were cryo-protected by briefly soaking in reservoir solution supplemented with $20 \%(\mathrm{v} / \mathrm{v})$ glycerol and then flash-cooled in liquid nitrogen. All X-ray diffraction data were collected at Shanghai Synchrotron Radiation Facility (SSRF) BL17U. The datasets were indexed, integrated, and scaled using HKL2000 ${ }^{34}$. The structures of variant RBD-hACE2 were determined via molecular replacement method using Phaser ${ }^{35}$ with the previously reported structures of SARS-CoV-2 RBD-hACE2 (PDB: 6LZG) as a search model. The atomic models were built using Coot 0.8.2 $2^{36}$ and the refinements were completed using Phenix.refine ${ }^{37}$. MolProbity was used to assess the stereochemical quality of the final models ${ }^{38}$. The data collection, processing, and refinement statistics were summarized in Supplementary Table 1. All structural figures were generated using the PyMOL 4.5 software (https://pymol.org/2/).

Surface plasmon resonance (SPR) assay. The SPR assays were performed to test the interactions between mFc-fused ACE2 (including hACE2 and miACE2) and SARS-CoV-2 variant RBDs using a BIAcore $8 \mathrm{~K}$ (GE Healthcare) with a CM5 chip (GE Healthcare) at $25^{\circ} \mathrm{C}$ in single-cycle mode. SARS-CoV-2 WT RBD was used as a positive control. The buffer system was PBST (10 mM Na2HPO4, $2 \mathrm{mM}$ $\mathrm{KH} 2 \mathrm{PO} 4, \mathrm{pH} 7.4,137 \mathrm{mM} \mathrm{NaCl}, 2.7 \mathrm{mM} \mathrm{KCl}, 0.005 \%$ Tween 20) and the antimIgG antibody (Cytiva) was pre-immobilized on the CM5 chip using standard amine coupling chemistry with a $50 \mu \mathrm{g} / \mathrm{mL}$ concentration. Concentrated supernatant containing hACE2 $-\mathrm{mFc}$ or miACE2 $-\mathrm{mFc}$ protein was captured on the chip using this immobilized antibody. Serially diluted WT RBD $(12.5,25,50,100$, $200 \mathrm{nM})$, Alpha RBD and Mink-Y453F RBD (3.125, 6.25, 12.5, 25, $50 \mathrm{nM})$, Beta RBD, Gamma RBD, and Mink-N501T RBD $(6.25,12.5,25,50,100 \mathrm{nM})$, MinkF486L RBD $(25,50,100,200,400 \mathrm{nM}), \operatorname{RBD} \mathrm{K} 417 \mathrm{~N}$ and RBD K417T $(20,40,80$, 160, $320 \mathrm{nM})$, RBD N501Y/E484K $(0.5,1,2,4,8 \mathrm{nM})$, RBD N501Y/K417N $(4,8$ 16, 32, $64 \mathrm{nM}), \mathrm{RBD}$ N501Y/K417T $(2,4,8,16,32 \mathrm{nM})$, and RBD E484K (5, 10, 20 $40,80 \mathrm{nM}$ ) were flowed over the chip to evaluate hACE2 binding. Various concentrations of WT RBD $(2.5,5,10,20,40 \mu \mathrm{M})$, Mink-Y453F RBD $(40,80,160,320$, $640 \mathrm{nM})$, Mink-F486L RBD (160, 320, 640, 1280, $2560 \mathrm{nM})$, and Mink-N501T RBD $(320,640,1280,2560,5120 \mathrm{nM})$ were also used to evaluate miACE2 binding The chip was regenerated after each reaction using glycine ( $\mathrm{pH}$ 1.7). The equilibrium dissociation constants $\left(K_{\mathrm{D}}\right)$ of each pair of RBD-hACE2 interaction and RBD-miACE2 interaction were analyzed using a 1:1 binding model and steady state affinity model in the Biacore Insight Evaluation 2.0.15.12933 software (GE Healthcare), respectively. These results were then visualized using Graphpad Prism 8.

Flow cytometry analysis. These assays used a stable BHK21 cell line expressing hACE2 which was first constructed. Briefly, lentiviruses were packaged in HEK293T cells co-transfected with pLV-C-GFPSpark-hACE2 (Sino Biological) and helper plasmid pLP1, pLP2, and pLP/VSV-G (Invitrogen) at a ratio of 20:20:13:5 using Lipofectamine 2000. After $72 \mathrm{~h}$, the supernatants containing the hACE2 lentiviruses were collected and used to infect BHK21 cells. GFP-positive cells were selected using a BD FACSAriaIII (Becton, Dickinson and Company) and separated into 96-well cell culture plates. A single positive colony was then picked for further culture. The cells were stored in liquid nitrogen following two times of cell sorting. The hACE2-expressing cells were grown for approximately 12 passages, and incubated with Phosphate Buffer Saline (PBS) or $2 \mu \mathrm{g} / \mathrm{mL}$ RBD protein (including MERS-CoV RBD, SARS-CoV-2 WT RBD, Alpha RBD, Beta RBD, Gamma RBD, Mink-Y453F RBD, Mink-N501T RBD, and Mink-F486L RBD) at $37^{\circ} \mathrm{C}$ for $30 \mathrm{~min}$. MERS-CoV RBD was used as a negative control. After washing with PBS, the cells 
were stained with APC anti-His tag antibody (1:500; BioLegend) at $37^{\circ} \mathrm{C}$ for $30 \mathrm{~min}$. These cells were then washed and resuspended in $200 \mu \mathrm{L}$ PBS before being evaluated using a BD FACSCanto II (Becton, Dickinson and Company). The percentage of RBD-binding cells can be described as the ratio of RBD-binding cells (Q2) to hACE2-positive cells (Q2 and Q3). Each group comprised at least three replicates and the FACS graphics were generated using FlowJo V7.6 software. Statistical analysis was performed using Graphpad Prism 8.

Molecular dynamics (MD) simulations. Briefly, both the WT RBD-hACE2 (PDB: 6LZG) and Mink-Y453F RBD-hACE2 structures were stripped of their N-acetyl- $\beta$ glucosaminide glycans and crystal structural waters and then used in ten parallel molecular dynamics $(\mathrm{MD})$ simulations with different random seeds. All MD simulations were performed using GROMACS (version 2020.5) ${ }^{39}$ on GPU with the CHARMM36 protein force field and TIP3P water model ${ }^{40,41}$. All calculations were applied using an atom-based truncation scheme and updated heuristically with a list cutoff of $12 \AA$, a non-bond cutoff of $12 \AA$, and a force switching function initiated at $10 \AA$ for Van der Waals interactions. Long-range electrostatic interactions were computed using Particle Mesh Ewald method with fourth-order cubic interpolation and $1.6 \AA$ grid spacing ${ }^{42}$. To equilibrate solvent molecules around the solute, each system was minimized using the steepest descent algorithm with a maximum force of $<1000 \mathrm{~kJ} / \mathrm{mol} / \mathrm{nm}$, followed by $200 \mathrm{ps}$ of NVT MD equilibration and $200 \mathrm{ps}$ of NPT equilibration with $1000 \mathrm{~kJ} / \mathrm{mol} \cdot \mathrm{nm} 2$ harmonic positional restraints on the heavy atoms of the protein, and $200 \mathrm{ps}$ NPT with $1000 \mathrm{~kJ} /$ mol.nm2 harmonic positional restraints on the protein backbone heavy atoms, and 200 ps NPT with reduced harmonic positional restraints of $500 \mathrm{~kJ} / \mathrm{mol}^{-\mathrm{nm}^{2}}$ on the protein backbone heavy atoms. Each equilibrated system was finally subjected to $100 \mathrm{~ns}$ NPT at $300 \mathrm{~K}$ using a Velocity rescaling thermostat without any restraints on the protein atoms ${ }^{43}$. During MD simulation, the LINCS algorithm ${ }^{44}$ used to constrain all of the bonds which rely on hydrogen atoms using a time step of $2 \mathrm{fs}$, and water molecules were restrained using the SETTLE algorithm ${ }^{45}$.

\section{Molecular mechanics/Poisson-Boltzmann surface area (MM/PBSA) binding energy calculations. For each WT RBD-hACE2 and Mink-Y453F RBD-hACE2} complex, 100 snapshots were extracted from the last $20 \mathrm{~ns}$ trajectories. A total of 1000 snapshots were combined and subjected to MM/PBSA calculations using the g_mmpbsa tool in GROMACS ${ }^{46}$. The solute dielectric constant was set to 2 and the entropy contribution was ignored. The binding energy was calculated as $\Delta \mathrm{E}_{\text {Bind- }}$ ing $=$ ERBD-hACE2 $-\left(E_{\text {RBD }}+E_{\text {hACE2 }}\right)$. The energy components consisted of the molecular mechanics $(\mathrm{MM})$ potential energy $\mathrm{E}_{\mathrm{MM}}$, and the solvation energy $\mathrm{E}_{\text {solvation. }}$.

Production and quantification of pseudoviruses. Pseudoviruses containing SARS-CoV-2 variant $\mathrm{S}$ protein and the backbone of deficient vesicular stomatitis virus (VSV) vector (VSV- $\Delta$ G-GFP) (BrainVTA) were generated using the previously described protocols ${ }^{47,48}$. In brief, $30 \mu \mathrm{g}$ of $\mathrm{S}$ plasmid with a $\mathrm{C}$ terminal 18 amino acids truncation was transfected into HEK293T cells cultured in a $10 \mathrm{~cm}$ dish, then after $24 \mathrm{~h}$ the VSV- $\Delta \mathrm{G}$-GFP pseudoviruses were added into the cell supernatant. The inoculum was then removed following incubation at $37^{\circ} \mathrm{C}$ for $2 \mathrm{~h}$ and the cells were washed with PBS and cultured in DMEM supplemented with both $10 \%$ FBS and anti-VSV-G antibody (produced by I1Hybridoma ATCC $\left.\mathrm{CRL}^{2} 700^{\mathrm{m}}\right)$. Then $20 \mathrm{~h}$ post-infection, the supernatants were harvested, filtered $\left(0.45 \mu \mathrm{m}\right.$ filter, Millipore, Cat\#SLHP033RB), aliquoted, and stored at $-80^{\circ} \mathrm{C}$.

Prior to quantification, the unpackaged RNA in the SARS-CoV-2 pseudoviruses was removed using a $0.5 \mathrm{U} / \mu \mathrm{L}$ BaseMuncher endonuclease (Abcam) treatment at $37^{\circ} \mathrm{C}$ for $1.5 \mathrm{~h}$. Viral RNA was extracted using an RNA extraction kit (Bioer Technology) and quantified using a quantitative RT-PCR assay performed using a 7500 Fast Real-Time PCR system (Applied Biosystems). The primers and probe used to detect the L gene of the VSV virus are as described in the literature ${ }^{49}$ : VSVF: TGATACAGTACAATTATTTTGGGAC; VSV-R: GAGACTTTCTGTTACGG GATCTGG; VSV-probe: FAM-ATGATGCATGATCCWGC-TAMRA.

Pseudovirus infection assays. The pseudovirus particles for SARS-CoV-2 and its variants were normalized and then diluted to the same amount using quantitative RT-PCR. Then $100 \mu \mathrm{L}$ of each pseudovirus was added to the cultured Huh7 cells. $15 \mathrm{~h}$ later, the plates were imaged and the number of infected cells, positive for GFP, was determined using a BD FACSCanto II. Each group contained at least three replicates. Statistical analysis was performed using Graphpad Prism 8.

Pseudovirus neutralization assays. Serially diluted hACE2 proteins (The maximum concentration was $240 \mu \mathrm{g} / \mathrm{mL}$ ) were incubated with SARS-CoV-2 or its variants at $37^{\circ} \mathrm{C}$ for $1 \mathrm{~h}$. Then the mixtures were added into the Vero cells in the 96-well plates. After $15 \mathrm{~h}$, the number of infected cells was analyzed using CQ1 confocal quantitative image cytometer (Yokogawa). Each group contained at least three replicates. The $\mathrm{EC}_{50}$ values were calculated using GraphPad Prism 8.

Statistic of SARS-CoV-2 variants $\mathbf{S}$ sequences. SARS-CoV-2 S protein sequences were downloaded from the GISAID Initiative database (gisaid.org) ${ }^{50}$ with the accession ID of the newest sequence submitted to the GISAID Initiative at the time of evaluation being EPI_ISL_1159228. We discarded the sequences (a total of 168,190$)$ that contain sequencing errors (including any sequences with " $\mathrm{X}$ " in the sequence) or inexact collection time. This left 506,858 sequences which were then individually aligned with reference Wuhan-Hu-1 S sequence (GISAID: EPI_ISL_402119) using Mafft v7.310 ${ }^{51}$. The S protein sequences with the fewest mutations in each SARS-CoV-2 variant were selected for further statistical analysis. Based on the collection time, the cumulative weekly number of new sequences was calculated for the period between April 20, 2020 and March 1, 2021. For MinkY453F, Mink-F486L, and Mink-N501T, the number of new sequences isolated in humans or minks were calculated individually. The frequency was calculated as the ratio of the cumulative number of each variant to the total cumulative number of sequences.

Reporting summary. Further information on research design is available in the Nature Research Reporting Summary linked to this article.

\section{Data availability}

The accession numbers for the atomic coordinates and diffraction data reported in this paper are PDB: 7EKF [https://doi.org/10.2210/pdb7EKF/pdb] (Structure of SARS-CoV-2 Alpha variant spike receptor-binding domain complexed with human ACE2), 7EKG [https://doi.org/10.2210/pdb7EKG/pdb] (Structure of SARS-CoV-2 Beta variant spike receptor-binding domain complexed with human ACE2), 7EKC [https://doi.org/10.2210/ $\mathrm{pdb} 7 \mathrm{EKC} / \mathrm{pdb}$ ] (Structure of SARS-CoV-2 Gamma variant spike receptor-binding domain complexed with human ACE2), 7EKH [https://doi.org/10.2210/pdb7EKH/pdb] (Structure of SARS-CoV-2 spike receptor-binding domain Y453F mutation complexed with human ACE2) and 7EKE [https://doi.org/10.2210/pdb7EKE/pdb] (Structure of SARS-CoV-2 spike receptor-binding domain F486L mutation complexed with human ACE2). Source data are provided with this paper.

Received: 5 May 2021; Accepted: 30 September 2021; Published online: 20 October 2021

\section{References}

1. Zhu, N. et al. A novel coronavirus from patients with pneumonia in China, 2019. N. Engl. J. Med. 382, 727-733 (2020).

2. Wang, C., Horby, P. W., Hayden, F. G. \& Gao, G. F. A novel coronavirus outbreak of global health concern. Lancet 395, 470-473 (2020).

3. Zhou, P. et al. A pneumonia outbreak associated with a new coronavirus of probable bat origin. Nature 579, 270-273 (2020).

4. Wang, Q. et al. Structural and functional basis of SARS-CoV-2 entry by using human ACE2. Cell 181, 894-904 e899 (2020).

5. Korber, B. et al. Tracking changes in SARS-CoV-2 spike: evidence that D614G increases infectivity of the COVID-19 virus. Cell 182, 812-827 e819 (2020).

6. Rambaut, A. et al. Preliminary genomic characterisation of an emergent SARS-CoV-2 lineage in the UK defined by a novel set of spike mutations. https://virological.org/t/preliminary-genomic-characterisation-of-anemergent-sars-cov-2-lineage-in-the-uk-defined-by-a-novel-set-of-spikemutations/563 (2020)

7. Leung, K., Shum, M. H., Leung, G. M., Lam, T. T. \& Wu, J. T. Early transmissibility assessment of the N501Y mutant strains of SARS-CoV-2 in the United Kingdom, October to November 2020. Euro Surveill 26, https:// doi.org/10.2807/1560-7917.ES.2020.26.1.2002106 (2021).

8. Davies, N. G. et al. Estimated transmissibility and impact of SARS-CoV-2 lineage B.1.1.7 in England. Science, eabg3055, https://doi.org/10.1126/ science.abg3055 (2021).

9. Tegally, H. et al. Emergence and rapid spread of a new severe acute respiratory syndrome-related coronavirus 2 (SARS-CoV-2) lineage with multiple spike mutations in South Africa. medRxiv, 2020.2012.2021.20248640, https:// doi.org/10.1101/2020.12.21.20248640 (2020).

10. Faria, N. R. et al. Genomic characterisation of an emergent SARS-CoV-2 lineage in Manaus: preliminary findings. https://virological.org/t/genomiccharacterisation-of-an-emergent-sars-cov-2-lineage-in-manaus-preliminaryfindings/586 (2021).

11. Fujino, T. et al. Novel SARS-CoV-2 variant identified in travelers from Brazil to Japan. Emerg Infect Dis 27, https://doi.org/10.3201/eid2704.210138 (2021)

12. Mallapaty, S. COVID mink analysis shows mutations are not dangerous - yet. Nature 587, 340-341 (2020)

13. Hammer, A. S. et al. SARS-CoV-2 transmission between mink (Neovison vison) and humans, Denmark. Emerg. Infect. Dis. 27, 547-551 (2021).

14. Oude Munnink, B. B. et al. Transmission of SARS-CoV-2 on mink farms between humans and mink and back to humans. Science 371, 172-177 (2021).

15. Lan, J. et al. Structure of the SARS-CoV-2 spike receptor-binding domain bound to the ACE2 receptor. Nature 581, 215-220 (2020).

16. Wu, L. et al. Broad host range of SARS-CoV-2 and the molecular basis for SARS-CoV-2 binding to cat ACE2. Cell Disco. 6, 68 (2020). 
17. Wu, L. et al. Molecular basis of pangolin ACE2 engaged by COVID-19 virus. Chin. Sci. Bull. 66, 73 (2021).

18. Molenaar et al. Clinical and pathological findings in SARS-CoV-2 disease outbreaks in farmed mink (Neovison vison). Vet. Pathol. 57, 653-657 (2020).

19. Zhu, X. et al. Cryo-electron microscopy structures of the N501Y SARS-CoV-2 spike protein in complex with ACE2 and 2 potent neutralizing antibodies. PLoS Biol. 19, e3001237 (2021).

20. Bayarri-Olmos, R. et al. The SARS-CoV-2 Y453F mink variant displays a striking increase in ACE-2 affinity but does not challenge antibody neutralization. bioRxiv https://doi.org/10.1101/2021.01.29.428834 (2021).

21. Starr, T. N. et al. Deep mutational scanning of SARS-CoV-2 receptor binding domain reveals constraints on folding and ACE2 binding. Cell 182, 1295-1310.e1220 (2020).

22. Garcia-Beltran, W. F. et al. Multiple SARS-CoV-2 variants escape neutralization by vaccine-induced humoral immunity. Cell https://doi.org/ 10.1016/j.cell.2021.03.013 (2021).

23. Hoffmann, M. et al. SARS-CoV-2 variants B.1.351 and P.1 escape from neutralizing antibodies. Cell https://doi.org/10.1016/j.cell.2021.03.036 (2021)

24. Yan, R. et al. Structural basis for the recognition of SARS-CoV-2 by full-length human ACE2. Science 367, 1444-1448 (2020).

25. $\mathrm{Gu}, \mathrm{H}$. et al. Adaptation of SARS-CoV-2 in $\mathrm{BALB} / \mathrm{c}$ mice for testing vaccine efficacy. Science 369, 1603-1607 (2020).

26. Ozono, S. et al. SARS-CoV-2 D614G spike mutation increases entry efficiency with enhanced ACE2-binding affinity. Nat. Commun. 12, 848 (2021).

27. Saito, A. et al. SARS-CoV-2 spike P681R mutation, a hallmark of the Delta variant, enhances viral fusogenicity and pathogenicity. bioRxiv, 2021.2006.2017.448820, https://doi.org/10.1101/2021.06.17.448820 (2021).

28. European Food Safety, A. et al. Monitoring of SARS-CoV-2 infection in mustelids. EFSA J. 19, e06459 (2021).

29. Cele, S. et al. Escape of SARS-CoV-2 501Y.V2 from neutralization by convalescent plasma. Nature, https://doi.org/10.1038/s41586-021-03471-w (2021).

30. Wibmer, C. K. et al. SARS-CoV-2 501Y.V2 escapes neutralization by South African COVID-19 donor plasma. Nat. Med. https://doi.org/10.1038/s41591021-01285-x (2021).

31. Monteil, V. et al. Inhibition of SARS-CoV-2 infections in engineered human tissues using clinical-grade soluble human ACE2. Cell 181, 905-913 e907 (2020).

32. Khan, A. et al. A pilot clinical trial of recombinant human angiotensin-converting enzyme 2 in acute respiratory distress syndrome. Crit. Care 21, 234 (2017).

33. Haschke, M. et al. Pharmacokinetics and pharmacodynamics of recombinant human angiotensin-converting enzyme 2 in healthy human subjects. Clin. Pharmacokinet. 52, 783-792 (2013).

34. Otwinowski, Z. \& Minor, W. [20] Processing of X-ray diffraction data collected in oscillation mode. Methods Enzymol. 276, 307-326 (1997).

35. Read, R. J. Pushing the boundaries of molecular replacement with maximum likelihood. Acta Crystallogr. D. Biol. Crystallogr. 57, 1373-1382 (2001).

36. Emsley, P. \& Cowtan, K. Coot: model-building tools for molecular graphics. Acta Crystallogr. D. Biol. Crystallogr. 60, 2126-2132 (2004).

37. Adams, P. D. et al. PHENIX: a comprehensive Python-based system for macromolecular structure solution. Acta Crystallogr. D. Biol. Crystallogr. 66, 213-221 (2010).

38. Chen, V. B. et al. MolProbity: all-atom structure validation for macromolecular crystallography. Acta Crystallogr. D. Biol. Crystallogr. 66, 12-21 (2010).

39. Abraham, M. J. et al. GROMACS: high performance molecular simulations through multi-level parallelism from laptops to supercomputers. SoftwareX 1-2, 19-25 (2015).

40. Jorgensen, W., Chandrasekhar, J., Madura, J., Impey, R. \& Klein, M. Comparison of simple potential functions for simulating liquid water. J. Chem. Phys. 79, 926-935 (1983).

41. Best, R. B. et al. Optimization of the additive CHARMM all-atom protein force field targeting improved sampling of the backbone phi, psi and sidechain chi(1) and chi(2) dihedral angles. J. Chem. Theory Comput. 8, 3257-3273 (2012).

42. Darden, T., York, D. \& Pedersen, L. Particle mesh Ewald: an N.log(N) method for Ewald sums in large systems. J. Chem. Phys. 98, 10089-10092 (1993).

43. Bussi, G., Donadio, D. \& Parrinello, M. Canonical sampling through velocity rescaling. J. Chem. Phys. 126, 014101 (2007).

44. Hess, B., Bekker, H., Berendsen, H. J. C. \& Fraaije, J. G. E. M. LINCS: a linear constraint solver for molecular simulations. J. Computational Chem. 18, 1463-1472 (1997).

45. Miyamoto, S. \& Kollman, P. A. Settle: an analytical version of the SHAKE and RATTLE algorithm for rigid water models. J. Computational Chem. 13, 952-962 (1992).

46. Kumari, R., Kumar, R., Open Source Drug Discovery Condortium \& Lynn, A. g_mmpbsa-a GROMACS tool for high-throughput MM-PBSA calculations. $J$. Chem. Inf. Model 54, 1951-1962 (2014).
47. Li, Q. et al. The impact of mutations in SARS-CoV-2 spike on viral infectivity and antigenicity. Cell 182, 1284-1294 e1289 (2020).

48. Muik, A. et al. Neutralization of SARS-CoV-2 lineage B.1.1.7 pseudovirus by BNT162b2 vaccine-elicited human sera. Science https://doi.org/10.1126/ science.abg6105 (2021).

49. Hole, K., Velazquez-Salinas, L. \& Clavijo, A. Improvement and optimization of a multiplex real-time reverse transcription polymerase chain reaction assay for the detection and typing of Vesicular stomatitis virus. J. Vet. Diagn. Invest. 22, 428-433 (2010).

50. Elbe, S. \& Buckland-Merrett, G. Data, disease and diplomacy: GISAID's innovative contribution global health. Glob. Chall. 1, 33-46 (2017).

51. Katoh, K., Misawa, K., Kuma, K. \& Miyata, T. MAFFT: a novel method for rapid multiple sequence alignment based on fast Fourier transform. Nucleic Acids Res. 30, 3059-3066 (2002).

\section{Acknowledgements}

We thank the staff of BL17U beamlines at Shanghai Synchrotron Radiation Facility. We are grateful to Yuanyuan Chen and Zhenwei Yang from Institute of Biophysics Chinese Academy of Sciences (CAS) and Zheng Fan from Institute of Microbiology CAS for technical assistance with SPR experiments. We thank the CQ1 confocal image cytometer from Yokogawa Electric Corporation. This work was supported by National Key Research and Development Program of China (2021YFC0863300), the Strategic Priority Research Program of the Chinese Academy of Sciences (XDB29010202, XDB37030204), the intramural special grant for SARS-CoV-2 research from the Chinese Academy of Sciences (CAS), National Key R\&D Program of China (2018YFC1200600), and National Natural Science Foundation of China (NSFC, 81922044 and 31800775). Q.H.W. is supported by the Youth Innovation Promotion Association CAS (2018119). G.F.G. is supported by the foundation of the NSFC Innovative Research Group (81621091).

\section{Author contributions}

Q.H.W., J.Q., and G.F.G. initiated and coordinated the project. Q.H.W., X.Z., J.Q., and G.F.G. designed the experiments. P.H., A.Z., and X.Z. performed the pseudovirus infection. Y.F.Z. and Q.C. completed the FACS. C.S. and S.N. conducted the SPR. P.H. C.S., Y.F.Z., C.Q., Y.Q.Z., W.L., H.L., J.L., Z.Z., C.B., and Y.H. expressed and purified proteins. Q.W. and N.H. performed MD and MM/PBSA binding energy calculations. P.H., C.S., and J.Q. collected the diffraction data and determined the complex structure P.H., C.S., and Q.H.W. analyzed the data and wrote the paper. H.C., M.Y., and J.Y. Q.H.W., X.Z., J.X.Q., G.F.G., participated in the paper-editing and discussion.

\section{Competing interests}

The authors declare no competing interests.

\section{Additional information}

Supplementary information The online version contains supplementary material available at https://doi.org/10.1038/s41467-021-26401-w.

Correspondence and requests for materials should be addressed to Xin Zhao, George Fu Gao or Jianxun Qi.

Peer review information Nature Communications thanks the anonymous reviewer(s) for their contribution to the peer review of this work.

Reprints and permission information is available at http://www.nature.com/reprints

Publisher's note Springer Nature remains neutral with regard to jurisdictional claims in published maps and institutional affiliations.

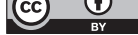

Open Access This article is licensed under a Creative Commons Attribution 4.0 International License, which permits use, sharing, adaptation, distribution and reproduction in any medium or format, as long as you give appropriate credit to the original author(s) and the source, provide a link to the Creative Commons license, and indicate if changes were made. The images or other third party material in this article are included in the article's Creative Commons license, unless indicated otherwise in a credit line to the material. If material is not included in the article's Creative Commons license and your intended use is not permitted by statutory regulation or exceeds the permitted use, you will need to obtain permission directly from the copyright holder. To view a copy of this license, visit http://creativecommons.org/ licenses/by/4.0/.

(C) The Author(s) 2021 